\title{
A girl with CLOVES syndrome with a recurrent PIK3CA somatic mutation and pancreatic steatosis
}

\author{
Hiroaki Hanafusa', Naoya Morisada ${ }^{1,2}$, Tadashi Nomura ${ }^{3}$, Daisuke Kobayashi', Yoshinobu Akasaka ${ }^{5}$, Ming Juan Ye ${ }^{2}$, \\ Kandai Nozu' ${ }^{2}$ Noriyuki Nishimura ${ }^{2}$, Kazumoto lijima ${ }^{2}$ and Hideto Nakao ${ }^{6}$
}

\begin{abstract}
CLOVES syndrome is characterized by congenital lipomatous overgrowth, vascular malformation, epidermal nevi, and scoliosis/spinal malformation. It is caused by somatic mosaicism of gain-of-function variants of PIK3CA. Here, we describe a novel case of a 5-year-old Japanese girl with CLOVES and concurrent pancreatic steatosis. She had a recurrent somatic mutation in PIK3CA (NM_006218.3: c.1357G >A, p.Glu453Lys), elevated HbA1c levels, and pancreatic steatosis. This case indicates that pancreatic screening is critical for PIK3CA-related disorders.
\end{abstract}

CLOVES syndrome (MIM \#612918) is a rare disorder, and the abbreviation stands for congenital lipomatous overgrowth, vascular malformation, epidermal nevi, and scoliosis/spinal malformation ${ }^{1}$. CLOVES syndrome is generally caused by somatic mosaicism of gain-offunction variants in PIK3CA (3q26.32). In a previous report ${ }^{2}$, mutant allele frequencies were found to vary in the affected tissues, although the mutation rate in peripheral blood was very low. Therefore, it is important to analyze DNA derived from affected tissues and not from the peripheral blood for the diagnosis of CLOVES syndrome.

Pancreatic steatosis (PS), also called pancreatic lipomatosis, is frequently found in the adult pancreas and is typically benign. PS is identified by histological analysis or imaging, e.g., hyperechogenicity on abdominal ultrasonography and hypodensity of the pancreas on computed tomography ${ }^{3}$. PS is associated with obesity, increased age, and diabetes mellitus $(\mathrm{DM})^{4}$. PS rarely

Correspondence: Naoya Morisada (morisada_kch@hp.pref.hyogo.jp)

${ }^{1}$ Department of Clinical Genetics, Hyogo Prefectural Children's Hospital, Kobe, Japan

${ }^{2}$ Department of Pediatrics, Kobe University Graduate School of Medicine, Kobe, Japan

Full list of author information is available at the end of the article. occurs during childhood, and some genetic disorders have been associated with the development of PS ${ }^{4}$. However, to the best of our knowledge, PS has not been reported in patients with CLOVES syndrome. In this report, we describe a pediatric patient with CLOVES syndrome with concurrent PS.

The patient was a 5-year-old Japanese girl who was the second child of nonconsanguineous healthy parents. At 26 weeks of gestation, she was diagnosed with left pleural effusion and fetal hydrops by ultrasonography, and pleural drainage was performed. She was delivered by emergency cesarean section at 33 weeks of gestation because of increasing fetal hydrops. Her Apgar score was $5(1 \mathrm{~min}) / 7$ ( $5 \mathrm{~min}$ ), and her birth weight was $2604 \mathrm{~g}$ (> 97th percentile). She showed scattered capillary malformations in the upper and lower lips and both upper and lower limbs and enlargement of both second toes at birth. At 5 years, she was referred to our outpatient department. At that time, she showed systemic lipomatous overgrowth, particularly in the thorax and legs. Ultrasonography revealed lipomatous overgrowth and no development of mammary gland tissue. Scoliosis was observed by spinal X-ray examination (Fig. 1a). Enlargement of both second toes was still observed. Brain magnetic resonance imaging showed no abnormality, and she had no developmental

\section{(c) The Author(s) 2019}

(c) (i) Open Access This article is licensed under a Creative Commons Attribution 4.0 International License, which permits use, sharing, adaptation, distribution and reproduction cc) in any medium or format, as long as you give appropriate credit to the original author(s) and the source, provide a link to the Creative Commons license, and indicate if changes were made. The images or other third party material in this article are included in the article's Creative Commons license, unless indicated otherwise in a credit line to the material. If material is not included in the article's Creative Commons license and your intended use is not permitted by statutory regulation or exceeds the permitted use, you will need to obtain permission directly from the copyright holder. To view a copy of this license, visit http://creativecommons.org/licenses/by/4.0/. 

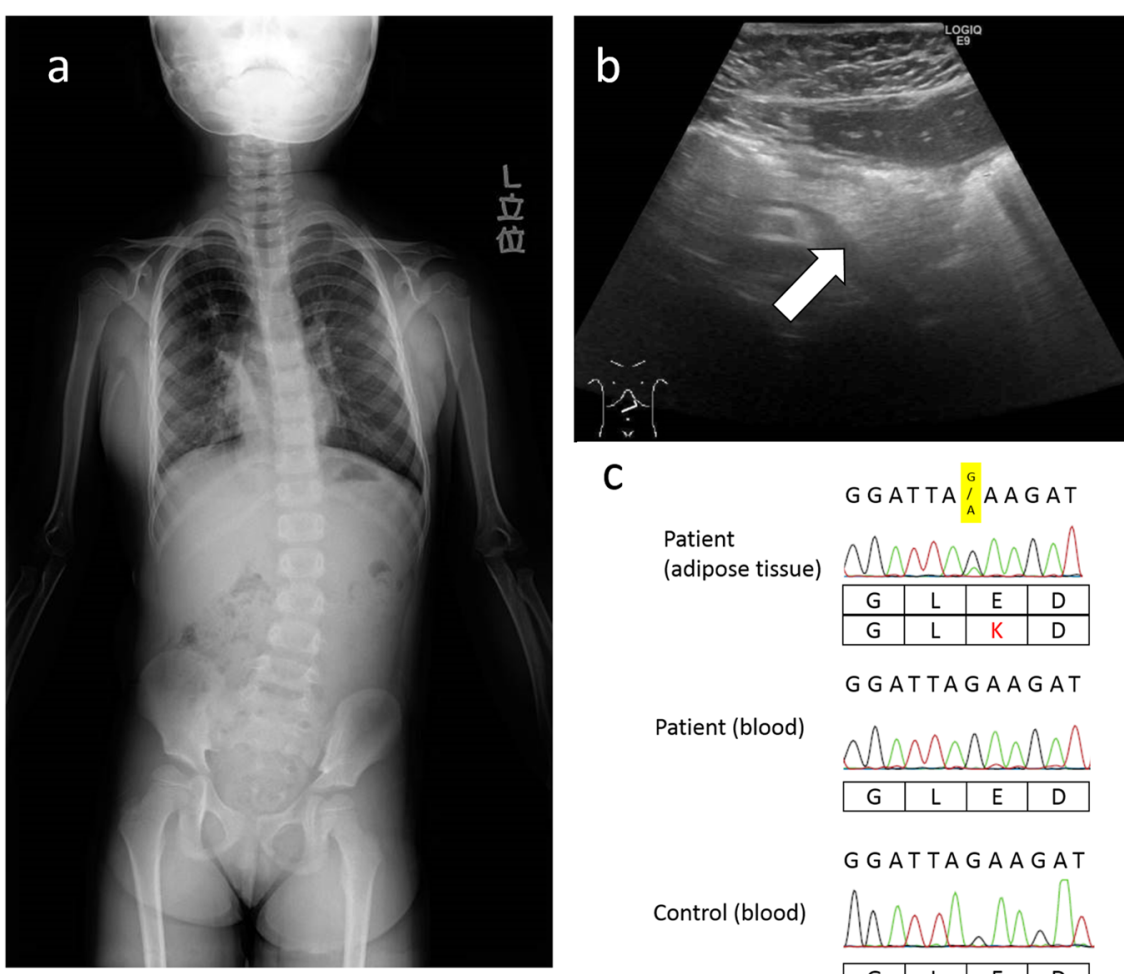

C

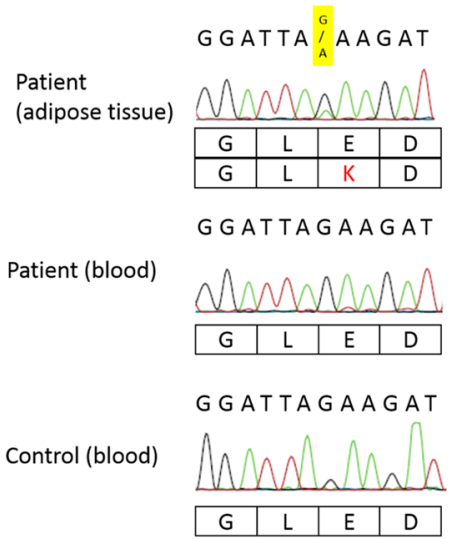

Fig. 1 a Chest radiograph of the patient showed scoliosis. b Ultrasonography showed a hyperechogenic pancreas (white arrow). c Results of PIK3CA gene analysis by Sanger sequencing. Identification of a mosaic missense variant of PIK3CA (c.1357G>A causing p.Glu453Lys) in DNA derived from skin fibroblasts; no variant was observed in DNA derived from peripheral blood

disorders. Accordingly, we considered a diagnosis of CLOVES syndrome. Abdominal ultrasonography showed pancreatic hyperechogenicity (Fig. 1b), indicating PS. Her hemoglobin A1c (HbA1c) was elevated (6.1\%). Her height at 5 years of age was $108.6 \mathrm{~cm}(-0.7 \mathrm{SD})$, and her body weight was $21.9 \mathrm{~kg}$ (body mass index, 18.6).

To confirm her molecular diagnosis, we analyzed DNA samples derived from peripheral blood and affected adipose tissues using Sanger sequencing to detect the PIK3CA variant after obtaining written informed consent from her parents. All procedures were reviewed and approved by the Institutional Review Board of the Kobe University School of Medicine and were performed in accordance with the ethical standards of the Declaration of Helsinki. We identified a heterozygous missense variant in PIK3CA (NM_006218.3: c.1357G>A, p.Glu453Lys) in DNA derived from affected adipose tissue, although the same variant was not found in DNA derived from peripheral blood (Fig. 1c). Mirzaa et al. ${ }^{5}$ reported four patients with c.1357G $>$ A diagnosed with megalencephalycapillary malformation-polymicrogyria (MCAP) syndrome or somatic overgrowth, including CLOVES syndrome. Finally, we diagnosed the patient with CLOVES syndrome. To consider the mosaic ratio, we performed droplet digital polymerase chain reaction $(\mathrm{ddPCR})^{6}$. We used $100 \mathrm{ng}$ of template DNA from adipose tissue and peripheral blood for ddPCR using a QX200 Droplet Generator, QX200 Droplet Reader, and QuantaSoft software (Bio-Rad, Hercules, CA, USA), and the mutant allele frequencies were found to be $29.7 \%$ and $0.27 \%$, respectively.

PIK3CA encodes a $110 \mathrm{kDa}$ catalytic subunit of phosphatidylinositol-3-kinase (PI3K). The PI3K/AKT/ mammalian target of rapamycin pathway is involved in cell proliferation and cell growth. Somatic PIK3CA variants cause abnormal activation of this pathway, and patients with PIK3CA aberration show overgrowth syndromes (e.g., CLOVES syndrome and MCAP syndrome). The umbrella term "PIK3CA-related overgrowth spectrum (PROS)" has been proposed ${ }^{7}$. Mirzaa et al. ${ }^{5}$ hypothesized that phenotypic differences in overgrowth syndromes caused by somatic mosaic PIK3CA variants may depend on the variant site and mosaic frequency. Piacitelli et al. $^{6}$ reported a patient with different mosaic ratios of PIK3CA p.Glu545Lys by tissue analysis and found no correlation between tissue-specific disease severity and mutant allele frequencies. We identified the mutant allele frequency of the patient to be $29.7 \%$ in affected tissue and $0.27 \%$ in white blood cells by ddPCR; hence, it is possible that mosaic frequency or differences 
in affected tissues might be involved. Further studies are required to explain these phenotypic differences.

The specific etiology of PS remains unclear. PIK3CA gain-of-function variants lead to overgrowth of fat tissues. Thus, patients with PROS are at risk of PS. Most patients with PS show a benign clinical course, but some may exhibit DM, pancreatic ductal failure, and pancreatic cancer. Our patient already showed elevated HbA1c levels, indicating that she was at high risk of DM and pancreatic cancer. Therefore, when clinicians encounter patients with PROS, pancreatic abnormalities should also be assessed.

In conclusion, our case demonstrated that pancreatic screening is essential for patients with PROS. Recently, specific therapy for PROS has been reported ${ }^{8}$; thus, it is necessary to establish approaches for the definitive diagnosis of PROS.

\section{HGV Database}

The relevant data from this Data Report are hosted at the Human Genome Variation Database at https://doi.org/10.6084/m9.figshare.hgv.2585.

\section{Acknowledgements}

We thank the patient and her family. We thank Editage (www.editage.jp) for English language editing.

\section{Author details}

${ }^{1}$ Department of Clinical Genetics, Hyogo Prefectural Children's Hospital, Kobe, Japan. ${ }^{2}$ Department of Pediatrics, Kobe University Graduate School of Medicine, Kobe, Japan. ${ }^{3}$ Department of Plastic Surgery, Kobe University Graduate School of Medicine, Kobe, Japan. ${ }^{4}$ Department of Orthopaedic Surgery, Hyogo Prefectural Children's Hospital, Kobe, Japan. ${ }^{5}$ Department of
Radiology, Hyogo Prefectural Children's Hospital, Kobe, Japan. ${ }^{6}$ Department of Neonatology, Hyogo Prefectural Children's Hospital, Kobe, Japan

\section{Conflict of interest}

The authors declare that they have no conflict of interest.

\section{Publisher's note}

Springer Nature remains neutral with regard to jurisdictional claims in published maps and institutional affiliations.

Received: 16 February 2019 Revised: 3 June 2019 Accepted: 7 June 2019. Published online: 24 June 2019

\section{References}

1. Sapp, J. C. et al. Newly delineated syndrome of congenital lipomatous overgrowth, vascular malformations, and epidermal nevi (CLOVE syndrome) in seven patients. Am. J. Med. Genet. A 143A, 2944-2958 (2007).

2. Kurek, K. C. et al. Somatic mosaic activating mutations in PIK3CA cause CLOVES syndrome. Am. J. Hum. Genet. 90, 1108-1115 (2012).

3. Coulier, B. Pancreatic lipomatosis: an extensive pictorial review. J. Belg. Soc. Radiol. 100, 39 (2016).

4. Smits, M. M. \& van Geenen, E. J. The clinical significance of pancreatic steatosis. Nat. Rev. Gastroenterol. Hepatol. 8, 169-177 (2011).

5. Mirzaa, G. et al. PIK3CA-associated developmental disorders exhibit distinct classes of mutations with variable expression and tissue distribution. JCI Insight 1, e87623 (2016)

6. Piacitelli, A. M. et al. Characterization of a severe case of PIK3CA-related overgrowth at autopsy by droplet digital polymerase chain reaction and report of PIK3CA sequencing in 22 patients. Am. J. Med. Genet. A 176 2301-2308 (2018).

7. Keppler-Noreuil, K. M. et al. PIK3CA-related overgrowth spectrum (PROS): diagnostic and testing eligibility criteria, differential diagnosis, and evaluation. Am. J. Med. Genet. A 167A, 287-295 (2015)

8. Venot, Q. et al. Targeted therapy in patients with PIK3CA-related overgrowth syndrome. Nature 558, 540-546 (2018). 